\title{
Effectiveness of dog rabies vaccination programmes: comparison of owner-charged and free vaccination campaigns
}

\author{
S. DURR ${ }^{1} \uparrow$, R. MINDEKEM ${ }^{2} \uparrow$, Y. KANINGA ${ }^{3}$, D. DOUMAGOUM MOTO ${ }^{2}$, \\ M. I. MELTZER ${ }^{4}$, P. VOUNATSOU ${ }^{1}$ AND J. ZINSSTAG ${ }^{1 *}$ \\ ${ }^{1}$ Swiss Tropical Institute, Basel, Switzerland \\ ${ }^{2}$ Centre de Support en Santé International, N’Djaména, Chad \\ ${ }^{3}$ Clinique Vétérinaire Urbaine, N'Djaména, Chad \\ ${ }^{4}$ Division of Emerging Infections and Surveillance Systems, Centers for Disease Control and Prevention, \\ Atlanta, USA
}

(Accepted 8 February 2009; first published online 30 March 2009)

\section{SUMMARY}

We investigated the percentage of dogs that could be vaccinated against rabies by conducting a pilot campaign in N'Djaména, Chad. Owners were charged US\$4.13 per dog vaccinated, and $24 \%$ of all dogs in the three city districts covered by the campaign were vaccinated. Total campaign costs were US\$7623, resulting in an average of US\$19.40 per vaccinated dog. This is five times more expensive than the cost per animal vaccinated during a previous free vaccination campaign for dog-owners, conducted in the same districts. The free campaign, which vaccinated 2605 more dogs than this campaign, cost an additional US\$1.45 per extra dog vaccinated.

Campaigns in which owners are charged for vaccinations result in lower vaccination rates than in free campaigns. Public health officials can use these results when evaluating the costs and benefits of subsidizing dog rabies vaccination programmes.

Key words: Canine rabies, Chad, owner charge, public good, vaccination.

\section{INTRODUCTION}

Rabies causes, worldwide, an estimated 55000 human deaths (90\% CI 24000-93000) per year. More than $99 \%$ of these deaths occur in developing countries, with about $43 \%$ (23750) occurring in Africa [1]. In countries where the virus circulates in the dog population, more than $99 \%$ of all human rabies cases are the result of exposure to rabid dogs [2, 3]. A person

\footnotetext{
* Address for correspondence: Professor J. Zinsstag, Swiss Tropical Institute, PO Box, CH-4002 Basel, Switzerland. (Email: jakob.zinsstag@unibas.ch)

$\dagger$ These authors contributed equally to this work.
}

bitten by a rabid dog, if untreated with post-exposure prophylaxis (PEP), has about a $5 \%$ (if bitten on hand) to $70 \%$ (if bitten on face) probability of developing clinical rabies [4, 5]. With one exception, clinical cases of human rabies are always fatal [6]. Unfortunately, PEP is often unavailable or unaffordable in many developing countries. Canine vaccination campaigns appear to be an effective means to control canine rabies [3]. Coleman \& Dye calculated that, to eliminate rabies from a dog population, a minimum of $39-57 \%$ of dogs must be vaccinated [7]. However, the World Health Organization (WHO) recommends that in order to eliminate dog rabies, vaccination coverage should reach $70 \%$ [3]. Examples include Japan (1957), 
Malaysia (1954), Taiwan (1961), Mexico (1990s) and several European countries, including elimination of wildlife rabies [8-11]. Recently, the USA declared itself to be free of dog-to-dog transmission of rabies (importation of rabid dogs remains a risk) [12].

However, many developing countries have no active official dog rabies control strategy. The current government policy on rabies control in Chad, for example, is restricted to human post-exposure treatment. Public human health authorities in Chad consider rabies a veterinary problem, and the Ministry of Livestock considers the problem to be 'negligible'. Human cases, however, are probably underreported [1]. Data from the incidence of dog bites in the United Republic of Tanzania, indicate that human rabies cases are between 10 and 100 times higher than officially reported [4]. In N'Djaména, capital of Chad, the annual incidence of canine rabies in 2006 was 1.7/ 1000 unvaccinated dogs [13]. In 2002 a pilot, free vaccination campaign for dog-owners in N'Djaména resulted in $64-87 \%$ of all dogs being vaccinated [14]. In that campaign, the societal costs (public sector costs + owner costs) were US\$3.11 per dog [15]. Kaare et al. obtained similar levels of coverage with a free vaccination campaign for dog-owners in agro-pastoralist communities in Tanzania [16]. Policy-makers can use cost and coverage data to both judge the value of, and plan necessary budgets for, dog rabies vaccination campaigns. Such cost data for Africa are scarce, with only three published cost per dog vaccination studies from sub-Saharan Africa [15-17]. We report in the present study the impact on dog rabies vaccination coverage when the owners are required to pay part of the costs of vaccination.

\section{MATERIAL AND METHODS}

N'Djaména's human population in 2001 was 649460 [18], with a dog population, as measured in a 2001 study, of 23560 dogs (95\% CI 14570-37 898). About $19 \%$ of the dogs were recorded as being vaccinated against rabies [19]. Our design for the dog vaccination campaign reported in the present study, conducted in October 2006, was similar to a pilot campaign in N'Djaména conducted in 2002 [14]. Location (two city districts), scope, advertising, equipment, operations, collaborators and (for the most part) the members of staff were the same as the previous campaign. The Chadian Ministry of Public Health gave written permission to conduct the study.

\section{Information campaign}

One week before the start of the vaccination campaign, the city government and the local chiefs of the two districts, in which the campaign was held, distributed posters announcing the campaign. Posters were displayed in the fronts of the houses of the local chiefs, at the vaccination points, and they were distributed to health centres and drug stores in the three districts. On the evening before the campaign started, we drove a car through the districts, using a megaphone to announce, in French, Arabic and Ngambaye, the date, locations and cost of the vaccinations. Advertisements about the campaign were transmitted four times by the local radio prior to the onset of the campaign. Finally, in some sections of the districts, the local chiefs went from door to door during the second day of the campaign to invite the dog-owners to come and have their dogs vaccinated.

\section{Charges to dog-owners and revolving fund}

The earlier free vaccination campaign for dog-owners cost US\$2.14 per vaccinated dog to the public sector, equivalent to FCFA 1110 [15]. For this campaign, owners were charged FCFA 2000 (US\$4.13) per dog vaccinated. This amount covered the campaign costs (assuming the same level of participation as in the earlier campaign), and included an amount intended to start a revolving fund. We envisioned that the revolving fund would be used to buy vaccine and equipment for future vaccination campaigns.

\section{Vaccination campaign}

The vaccination area was almost identical to the previous campaign and covered the sixth and seventh districts of N'Djaména, where $50-75 \%$ of all households have at least one dog [20]. As in the previous campaign, to aid logistics, we divided these two districts into three vaccination zones. The first zone was about $0.8 \mathrm{~km}^{2}$ and located in the seventh district. The second and third zones were in the sixth district, and were about $1.5 \mathrm{~km}^{2}$ and $1.3 \mathrm{~km}^{2}$, respectively.

Ten vaccination points were established in each vaccination zone. In each zone, the campaign took place during 3 days over a weekend (Friday-Sunday), $4 \mathrm{~h}$ per day with a break during lunchtime. Two veterinary technicians operated each vaccination point, and a local chief was also present. All veterinary technicians participated in a training day before the campaign and received (free) prophylactic anti-rabies 
vaccinations (0-7-21 vaccination scheme). Each point was equipped with 35 doses of vaccine (Rabisin ${ }^{\circledR}$, Merial, France), which were kept on ice in ice boxes, a register for recording the vaccinated animals, $50 \mathrm{syr}-$ inges and needles, 50 vaccination certificates, a cash box and receipt book, muzzles or cord to prevent dog bites, and collars and paint to mark the dog after vaccination. We did not provide more than 35 vaccine doses at the beginning of each working day in order to hold vaccine as long as possible in a fridge at a central location. Vaccination points were re-supplied as needed during the day. The local chief supplied table and chairs. Three people supervised the campaign, and they drove by car between the vaccination points to ensure the continuous supply of vaccine, syringes and certificates. In case of an accidental animal bite, a first-aid kit with was available to the supervisors.

We subcutaneously vaccinated dogs, cats and monkeys. For each animal, a new syringe and needle was used. If an owner was unable to bring an animal to a vaccination point, vaccination technicians went to the owner's house. We recorded owners' addresses, and the age, sex and colour of each vaccinated animal, which were marked by a collar and paint. The loss of collars and paint on marked dogs was estimated at 1.87 and $2 \cdot 2$ /day per $100 \mathrm{dogs}$, respectively (A. Gsell, unpublished data). Animals aged $<3$ months, and those obviously ill, were not vaccinated (vaccination is contra-indicated for these groups).

\section{Recapture of marked and unmarked dogs}

To assess vaccination coverage, we used the same capture-recapture methodology used by Kayali et al. [14]. Three observers walked through transect lines in each zone to detect all dogs in the street. There were 12 and 17 transect lines with a total distance of about $2.2 \mathrm{~km}$ and $3.3 \mathrm{~km}$ in the second and third zone, respectively. Detected dogs were counted, and a note was made as to whether or not they were marked with the campaign collar and/or paint, indicating vaccination. In each zone, the transect walks were done on the first and second days after the vaccination campaign. Each day, observers did two transects, one the morning and one the evening, when the dogs were most likely to be outside household compounds. The transect lines ran on parallel roads inside the vaccination zone, and we left a buffer zone of $\sim 220 \mathrm{~m}$ wide on the border of each zone to avoid counting dogs that migrated in, even temporarily, from outside the zone. To avoid double counting within a zone, the transect lines in each zone were separated by at least one street. Due to flooding following a torrential rain, we were unable to survey dogs in zone 1 .

\section{Household questionnaire}

In each zone, 3 days after the last vaccination day, we also interviewed the heads (if absent, a suitable substitute) of randomly selected households with at least one animal (dog, cat or monkey). The questionnaire was written in French, and, if necessary, the questions were translated in local languages for the interview. We asked the number of dogs owned by the household, if the owner had them vaccinated in the campaign, and reasons for non-participation in the campaign. For dogs unmarked during the campaign, but owneridentified as having been previously vaccinated, we checked the date on the vaccination certificate to see whether the vaccination was still valid ( $<1$ year old). To estimate the probability of confinement, we asked how much time the dogs spent outside the compound and recorded if, during the interview, the door was open. We collected 160 questionnaires from zone 1, 133 from zone 2 and 132 from zone 3. We assumed binomial distributions to calculate the statistical confidence intervals for each reported reason for nonparticipation.

\section{Analysis of the data for vaccination coverage}

To estimate the proportion of vaccinated dogs (owned and ownerless) in the whole dog population, we used a Bayesian (probability-based) mathematical model [14]. Appendix 1 (available online) describes the model in detail. All dogs counted in each survey were included in the mathematical model. We calculated the binomial probability distributions describing total owned dog population and the probabilities of confinement using data collected from the transect surveys and household questionnaires. The PetersonBailey formula was used to estimate the total owned dog population [21]. We assumed that confined (owned) dogs were not counted during the transect surveys and used the distribution from Kayali et al. [14] to estimate the number of ownerless dogs (see Appendix 1, online).

\section{Cost data}

We categorized cost data collected as either 'public sector' or 'private sector'. Public sector was defined 
as all costs that are paid using public funds, while private sector refers to costs paid by dog owners. From direct observation, we collected public-sector cost data for the following items: human rabies vaccine (prophylaxis for campaign workers), canine rabies vaccine, ice boxes with ice, syringes and needles (one of each per animal vaccinated), certificates, collars, cash boxes, receipts, muzzles, registers, pens, salaries (for campaign workers, local chiefs and supervisors), transport costs for supervision (car rental, petrol). We priced dog rabies vaccine at FCFA 425/ dose (US\$0.88), which was the price without a profit margin for the reseller in N'Djaména (the vaccine doses were donated by the manufacturing company; Merial). Public sector costs for the information campaign consisted of the costs of printing posters and running the radio advertisements.

For owner-related costs (private sector), we assumed an opportunity cost of labour of FCFA 500 (US\$1.04) for every owner presenting an animal for vaccination [15]. Animal owner transport costs were considered negligible because of the short distance from residences to the vaccination points in each zone.

\section{RESULTS}

\section{Characteristics of the population}

We visited 356 households with at least one animal in the three zones. The majority of the households kept one $\operatorname{dog}(78 \%)$ and $2 \%$ of the households had no dog (Table 1). The average age of the dogs was $3 \cdot 4$ years, and almost one fourth of the animals were aged $<1$ year. The majority $(77 \%)$ of all animals were male. From owner statements, at least $70 \%$ of the animals had been vaccinated against rabies at least once in their lives, but only $44 \%$ had a valid vaccination certificate $(\leqslant 1$ year old). Of those with a valid certificate, $61 \%$ had been vaccinated during the campaign (Table 1).

\section{Campaign operations and vaccination coverage achieved}

We successfully maintained the cold chain for the vaccine with ice boxes and ice, and were able to vaccinate all the animals brought to the vaccination points without any reports of animal bites. We vaccinated a total of 393 animals, of which 352 were dogs (102 in zone 1; 94 in zone 2; 156 in zone 3 ). We also vaccinated 11 cats, 12 monkeys and nine animals
Table 1. Characteristics of the 356 households and 450 animals surveyed in the vaccination zones*

\begin{tabular}{|c|c|c|}
\hline & $n$ & $\%$ \\
\hline \multicolumn{3}{|l|}{ Number of persons per household } \\
\hline $1-10$ & 230 & 65 \\
\hline $11-20$ & 107 & 30 \\
\hline $21-30$ & 10 & 3 \\
\hline $30-35$ & 1 & 0 \\
\hline Unknown & 8 & 2 \\
\hline \multicolumn{3}{|l|}{ Gender of interviewed respondents } \\
\hline Female & 165 & 46 \\
\hline Male & 190 & 53 \\
\hline Unknown & 1 & 1 \\
\hline \multicolumn{3}{|l|}{ Age (years) of interviewed respondents } \\
\hline \multicolumn{3}{|l|}{ Mean $(33 \cdot 7)$} \\
\hline \multicolumn{3}{|l|}{ Minimum $(13 \cdot 0)$} \\
\hline \multicolumn{3}{|l|}{ Maximum $(80 \cdot 0)$} \\
\hline \multicolumn{3}{|c|}{ Number of respective animals per household } \\
\hline \multicolumn{3}{|c|}{ Dogs } \\
\hline 0 & 7 & 2 \\
\hline 1 & 278 & 78 \\
\hline 2 & 65 & 18 \\
\hline 3 & 6 & 2 \\
\hline \multicolumn{3}{|l|}{ Cats } \\
\hline 0 & 341 & 96 \\
\hline 1 & 14 & 4 \\
\hline 2 & 1 & 0 \\
\hline \multicolumn{3}{|l|}{ Monkeys } \\
\hline 0 & 346 & 97 \\
\hline 1 & 10 & 3 \\
\hline \multicolumn{3}{|l|}{ Age (years) of animals } \\
\hline$<1$ & 101 & 22 \\
\hline $1-3$ & 155 & 34 \\
\hline $3-6$ & 134 & 30 \\
\hline$>6$ & 48 & 11 \\
\hline Unknown & 12 & 3 \\
\hline Average (years) & $3 \cdot 37$ & \\
\hline \multicolumn{3}{|l|}{ Animal gender } \\
\hline Male & 346 & 77 \\
\hline Female & 101 & 22 \\
\hline Unknown & 3 & 1 \\
\hline \multicolumn{3}{|c|}{ Animals stated to be vaccinated at least once in their lif } \\
\hline Yes & 314 & 70 \\
\hline With valid vaccination $\dagger$ & 197 & 44 \\
\hline Vaccinated during the campaign & 121 & 27 \\
\hline No & 132 & 29 \\
\hline Unknown & 4 & 1 \\
\hline
\end{tabular}

* Adapted from Dürr et al. [22].

$\uparrow$ For dogs unmarked during the campaign, but owneridentified as having been previously vaccinated, we checked the date on the vaccination certificate to see whether the vaccination was still valid ( $<1$ year old). These dogs would have been vaccinated before our campaign.

whose species was unidentified in our records. Most of the animals came from within the three vaccination zones, with 38 coming from outside those zones. 
Table 2. (a) Overall vaccination coverage and (b) vaccination coverage of owned dogs: owner-charged vs. free vaccination campaigns

\begin{tabular}{ccc}
\hline \hline & $\begin{array}{l}\text { Owner charged* } \\
\text { (new study) }\end{array}$ & $\begin{array}{l}\text { Free vaccination } \\
\text { (previous study) }\end{array}$ \\
\hline $\begin{array}{c}\text { (a) Overall vaccination coverage in all dogs } \\
\text { Zone 1 }\end{array}$ & - & $87 \%(84-89)$ \\
Zone 2 & $23 \%(0 \cdot 3-26)$ & $71 \%(64-76)$ \\
Zone 3 & $24 \%(0 \cdot 0-24)$ & $64 \%(58-71)$ \\
(b) Vaccination coverage only in owned dogs \\
Zone 1 & - & $88 \%(84-92)$ \\
Zone 2 & $27 \%(0 \cdot 0-27)$ & $76 \%(71-81)$ \\
Zone 3 & $24 \%(0 \cdot 0-24)$ & $70 \%(66-76)$ \\
\hline \hline
\end{tabular}

Values in parentheses are $95 \%$ confidence intervals.

* Due to flooding following a torrential rain, we were unable to survey dogs, and thus estimate vaccination coverage, in zone 1.

We estimated the owned-dog population to be $336(95 \%$ CI $323-349)$ in zone 2, and 581 (95\% CI $562-599$ ) in zone 3 . These numbers are similar (in terms of average number of dogs per household) to those estimated in the earlier campaign [14]. We also estimated that there were 84 ownerless dogs in zone 2 ( $25 \%$ of total zone population) and $87(1.5 \%$ of the total zone population) in zone 3 .

We achieved an overall dog vaccination coverage of $23 \%$ (95\% CI $0 \cdot 3-26)$ for zone 2 and $24 \%$ (95\% CI 0.0-24) for zone 3 (Table 2a). As mentioned previously, we were unable to collect population or vaccination coverage data from zone 1 .

\section{Reasons for non-participation}

Of the 328 respondents who did not have their dogs vaccinated, the two most common reasons cited for non-participation were not being informed $(26 \%$, $95 \%$ CI $21-30)$, and lack of money $(25 \%, 95 \%$ CI 20-30) (Table 3). Several respondents also stated that current household financial resources were spent on paying school fees (the school year began during the campaign). Ramadan, which coincided with the campaign, was also mentioned as a reason for nonparticipation (probably due to higher than normal food expenditures related to Ramadan). However, it should be noted that, in the districts where the campaign was conducted, the majority of persons were Christians.

Dog-related reasons for non-participation included: recent vaccination $18 \%$ (95\% CI 14-23); 'too young' (age $<3$ months) and/or ill or being unable to 'handle' the dog [these three reasons combined accounted for $9 \%(95 \%$ CI $6-12)$ of non-participating respondents]. Owner-specific reasons, such as lack of time, no interest or false information (e.g. expecting vaccinators to call door-to-door or thinking that rabies vaccination is only reasonable for aggressive dogs) accounted for $16 \%$ (95\% CI 12-20) of the stated reasons for non-participation (Table 3).

\section{Cost analysis}

The societal cost of the campaign totalled US\$7623 (public sector US\$5595; private/owner sector US\$2028), with the biggest single expense category being salaries (about $45 \%$ of societal costs) (Table 4 ). Owner payments accounted for $\sim 21 \%$ of societal costs, whilst vaccines, syringes, certificates, collars, and receipts only accounted for $6 \%$ of societal costs. The societal cost per vaccinated animal was US $\$ 19.40$ (public sector US\$14.24; private sector US\$5.16) (Table 4). This is about five times greater than the cost of US\$3.80 per animal vaccinated achieved during the free vaccination campaign for dog-owners (Table 4).

Although the earlier free vaccination campaign cost more than our campaign, it also resulted in more animals being vaccinated (i.e. greater public health impact) (Table 4). Compared to the campaign reported here, the earlier free vaccination campaign cost an additional US\$1.45 per extra animal vaccinated (societal perspective; incremental cost effectiveness ratio calculated as increased costs divided by the increase in number of animals vaccinated).

\section{DISCUSSION}

We conducted a vaccination campaign in which we charged owners a fee that covered the cost of the vaccine plus an amount meant to fund future campaigns. As far as we are aware, this is the first time, in a Sahelian country, that both the total societal costs and coverage rates have been recorded for a rabies vaccination campaign in which owners were charged a fee. The data from this study are essential elements when public health officials wish to assess the potential cost-effectiveness of subsidizing dog rabies vaccination programmes.

The campaign only achieved vaccination coverage rate of $\sim 25 \%$. This vaccination rate is well below the WHO's recommendation of $70 \%$. The overall vaccination coverage from this campaign was much lower than the $64-87 \%$ achieved in the earlier, free 
Table 3. Owner-stated reasons for non-participation in the campaign

\begin{tabular}{lcrc}
\hline \hline Reason & $\begin{array}{c}\text { Persons } \\
(n)\end{array}$ & $\begin{array}{c}\text { Previous } \\
\text { study }(\%)\end{array}$ \\
\hline Lack of money & 82 & 25 & - \\
Not informed & 84 & 26 & $11-26^{*}$ \\
Other owner-related reasons & 51 & 16 & \\
$\quad$ Lack of time & 15 & 5 & \\
No interest & 17 & 5 & \\
Expected a door-to-door campaign & 11 & 3 & \\
Thought vaccination only for aggressive dogs & 1 & 0 & \\
Against the campaign & 7 & 2 & \\
Dog-related reasons & 88 & 27 & $18-35 \dagger$ \\
Recently vaccinated & 60 & 18 & $10-17$ \\
Less than 3 months old & 12 & 4 & \\
Illness; 'too old' & 3 & 1 & \\
Dog not manageable & 13 & 4 & $19-35$ \\
Unknown reason & 23 & 7 & \\
Total & 328 & 100 & \\
\hline \hline
\end{tabular}

* Owner-specific reasons (not informed and lack of time).

$\dagger$ Dog-specific reasons (age, recent birth of puppies, illness, escape from home on vaccination day).

vaccination campaign (Table $2 a$ ). Even considering vaccination coverage in owned dogs, our campaign obtained coverage rates of $<30 \%$, which is less than half the rate achieved during the earlier free vaccination campaign (Table $2 b$ ). Because our campaign vaccinated far fewer animals than the previous free campaign, the cost per animal vaccinated in our campaign was about five times greater than in the free campaign. The free vaccination campaign vaccinated about nine times more animals than our campaign, yet the public-sector cost of the free campaign was only 1.5 times more than the current campaign. Although free campaigns are likely to be more effective (i.e. more animals vaccinated), the increased cost of free campaigns, especially to the public sector (Table 4), may present significant budgetary problems that could prevent the routine implementation of such campaigns. In Chad, in 2005, the government spent the equivalent of US\$9 per capita on health (calculated using standard exchange rates) [23].

The two main owner-stated reasons for not vaccinating dogs were lack of money and not having been informed of the campaign. In households with unvaccinated dogs, $25 \%$ of owners stated that lack of money was the reason for non-participation in the vaccination campaign. During the household survey we asked the owners a question regarding the fee that they would be willing to pay for vaccination [22]. To reach $70 \%$ vaccination coverage, a maximum of
FCFA 400-700 (US\$0.83-1.45) could be charged, which would not cover the vaccination campaign's cost [22].

Despite the pre-campaign efforts to inform owners, $26 \%$ of owners of unvaccinated dogs claimed not to have been informed about the campaign. Comparing the estimated number of dogs per zone and per households with our earlier study [14], we do not believe that the number of dogs changed 'notably' between the two campaigns. Since we achieved a $64-84 \%$ vaccination rate in the 2002 (free campaign for dog-owners) [14], the number of dogs that were not vaccinated because owners stated that they were 'not informed' was far greater in 2006 than in 2002. This higher proportion of owners claiming not to have been informed could be owners using this reason because they were uncomfortable about admitting the actual reason for non-participation, i.e. lack of money.

It is remarkable that only $4 \%$ of respondents indicated dog-handling difficulties as a reason for nonvaccination of the dog. In the previous free campaign, $19-35 \%$ of respondents indicated this was the reason for non-participation. Although only $3 \%$ of the interviewed persons indicated that they expected a door-to-door campaign, this method could be more effective in urban settings. However, in the previous free campaign, a high level of coverage was achieved with the vaccination point method. Ownerless dogs 
Table 4. Campaign cost comparisons: owner-charged vs. free vaccination

\begin{tabular}{|c|c|c|c|c|}
\hline & \multicolumn{2}{|c|}{ Owner charged (new study)* } & \multicolumn{2}{|c|}{ Free vaccination (previous study)* } \\
\hline & $\begin{array}{l}\text { Prices in } \\
\text { FCFA } †\end{array}$ & $\begin{array}{l}\% \text { of total } \\
\text { societal cost }\end{array}$ & $\begin{array}{l}\text { Prices in } \\
\text { FCFA } †\end{array}$ & $\begin{array}{l}\% \text { of total } \\
\text { societal cost }\end{array}$ \\
\hline \multicolumn{5}{|l|}{ Public sector } \\
\hline Vaccine, syringe, certificate, collar, receipt & 223460 & $0 \cdot 06$ & 1731218 & $0 \cdot 31$ \\
\hline Human pre-prophylaxis vaccination & 291700 & $0 \cdot 08$ & 291700 & $0 \cdot 05$ \\
\hline Equipment of vaccination point & 564291 & $0 \cdot 15$ & 368300 & $0 \cdot 07$ \\
\hline Salaries $\S$ & 1687200 & $0 \cdot 46$ & 897500 & $0 \cdot 16$ \\
\hline Transport & 404000 & $0 \cdot 11$ & 400000 & $0 \cdot 07$ \\
\hline Information & 326000 & $0 \cdot 09$ & 330000 & $0 \cdot 06$ \\
\hline Income from animal owners & -786000 & $-0 \cdot 21$ & 0 & $0 \cdot 00$ \\
\hline Total public sector & 2710651 & $0 \cdot 73$ & 4018718 & $0 \cdot 73$ \\
\hline \multicolumn{5}{|l|}{ Private sector } \\
\hline Working loss and transport & 196500 & $0 \cdot 05$ & 1500000 & $0 \cdot 27$ \\
\hline Vaccination cost & 786000 & $0 \cdot 21$ & 0 & $0 \cdot 00$ \\
\hline Total private sector & 982500 & $0 \cdot 27$ & 1500000 & $0 \cdot 27$ \\
\hline \multicolumn{5}{|l|}{ Societal cost } \\
\hline Total & 3693151 & & 5518718 & \\
\hline Number of animals vaccinated & 393 & & 3000 & \\
\hline \multicolumn{5}{|l|}{ Average cost per animal vaccinated } \\
\hline Public sector & 6897 & & 1340 & \\
\hline Private sector & 2500 & & 500 & \\
\hline Societal cost & 9397 & & 1840 & \\
\hline \multicolumn{5}{|l|}{ Total cost (US\$) } \\
\hline Public sector & 5595 & & 8295 & \\
\hline Private sector & 2028 & & 3096 & \\
\hline Societal cost & 7623 & & 11391 & \\
\hline \multicolumn{5}{|l|}{ Cost per animal vaccinated } \\
\hline Public sector & $14 \cdot 24$ & & $2 \cdot 76$ & \\
\hline Private sector & $5 \cdot 16$ & & $1 \cdot 03$ & \\
\hline Societal cost per vaccinated animal (US\$) & $19 \cdot 40$ & & $3 \cdot 80$ & \\
\hline $\begin{array}{l}\text { Incremental cost per extra animal vaccinated } \\
\text { in free vaccination campaign } \|\end{array}$ & $1 \cdot 45$ & & & \\
\hline
\end{tabular}

* Owner-charged campaign had 10 vaccination points and ran for a total of 9 days; free vaccination campaign had 8 vaccination points and ran for a total of 5 days.

$\uparrow 1000$ FCFA $=$ US $\$ 2.064$ (http://www.oanda.com/convert/classic).

$\$$ All 20 vaccination workers received three doses each.

$\S$ Daily cost per vaccination point 22440 FCFA in the owner-charged campaign, 18750 FCFA in the free vaccination campaign.

- Societal cost $=$ public cost + private cost.

\| Incremental cost-effectiveness ratio calculated as increased costs (costs in free campaign minus costs of owner-charged) divided by the increase in number of animals vaccinated (number vaccinated during free campaign minus number vaccinated during owner-charged campaign).

are inaccessible for parenteral vaccination, but they are estimated to be, on average, $<15 \%$ of the total dog population.

\section{Study limitations}

The biggest limitation of this study is that we were unable to link the costs of vaccination campaign to any human public health benefit. Indeed, with only $25 \%$ vaccination coverage, it is possible that this (second) campaign did not produce any reduction in risk of human rabies. Vaccination coverage was estimated by a Bayesian model (see Appendix 1, online), which explicitly considers uncertainties of questionnaire information such as dog confinement and the estimation of percentage ownerless dogs. 
Although the two campaigns were organized using the same principles and in the same districts, there were several differences between the two (e.g. in area covered, number of vaccination points, and duration of the campaigns). However, since the dog and human population densities, were about the same for both campaigns, the impact of the two campaigns can be directly compared. Another difference between the campaigns is that the 2006 campaign spent about twice the amount of the first campaign on salaries. The increased salaries were due to the different number of vaccination points and working days during the campaign (Table 4, second note). The equipment costs for the 2006 campaign were also greater than the previous campaign. Future campaigns have the potential of reducing costs by more closely monitoring the number of vaccination points, workers hired and salaries paid. Another limitation in extrapolating these results to future campaigns is that, in both campaigns, vaccine was valued 'at cost' (without a profit margin for the wholesalers). Vaccines may cost more in future campaigns.

This study, and the previous free vaccination study, each only measured the costs and impacts from one vaccination campaign. Since rabies in African countries is often endemic in animal populations other than dogs, and rabies vaccine provides timelimited protection ( $\sim 1$ year), there is always a danger of a rapid re-introduction of rabies in dog populations if vaccination rates fall below the WHO recommended rate of $70 \%$. Thus, in Africa, there is a need to have annual dog rabies vaccination campaigns to ensure the WHO recommendation is met. This study did not measure the costs associated with conducting routine annual vaccination campaigns.

Owner participation in this vaccination campaign may have been influenced by the previous free vaccination campaign that was held in the same area (albeit 4 years earlier). This earlier campaign could have increased (higher disease awareness or recognition of the benefits of vaccination) or decreased (people expect free vaccination) participation. We were unable to measure or account for such effects.

\section{CONCLUSIONS}

Dog vaccination is a feasible and comparatively cheap method to control rabies in endemic countries
$[15,24]$. However, this study showed that relying on campaigns in which owners are charged for each animal vaccinated will probably result in vaccination rates much lower than the WHO recommended rate of $70 \%$.

The results from this study can be used in future economic analyses examining the costs and benefits of dog vaccination campaigns. Such studies should explicitly take into account the reduction in human rabies cases resulting from increased dog rabies vaccination rates. The differences between this campaign and the previous campaign in fixed costs, such as salaries, transport and equipment, illustrate that it is essential that methods be developed to adequately control fixed costs. If fixed costs are not successfully controlled, then potentially cost-effective methods, such as 'free vaccination for dog-owners campaigns', of achieving vaccination coverage rates of $70 \%$ will not be affordable for government budgets. Without a political commitment, leading to rabies elimination, rabies will remain endemic in African cities, leaving millions of people in fear of exposure, and often without the hope of access to effective post-exposure treatment.

\section{APPENDIX A}

Table A1. Prior distribution for recapture and confinement probabilities in the two vaccination zones

\begin{tabular}{lcc}
\hline \hline Zone $1^{*}$ & Zone 2 & Zone 3 \\
\hline $\begin{array}{l}\text { Recapture probabilities } \\
p_{t}(i) \dagger\end{array}$ & $0 \cdot 056-0 \cdot 54$ & $0 \cdot 084-0 \cdot 54$ \\
Coverage $\left(C_{i}\right)$ & $0 \cdot 089-0 \cdot 60$ & $0 \cdot 133-0 \cdot 60$ \\
Encountering $\left(E_{i}\right)$ & $0 \cdot 70-0 \cdot 90$ & $0 \cdot 70-0 \cdot 90$ \\
Recording $\left(R_{i}\right)$ & $0 \cdot 90-0 \cdot 99$ & $0 \cdot 90-0 \cdot 99$ \\
Confinement of owned marked dogs, $c 1(i) \ddagger$ & \\
$\quad$ Beta $(\alpha, \beta)^{(i)}$ & $5 \cdot 554$ & \\
$\alpha$ & $13 \cdot 866$ & $14 \cdot 127$ \\
$\beta$ & & \\
Confinement of owned unmarked dogs, $c 2(i) \ddagger$ & \\
Beta $(\alpha, \beta)^{(i)}$ & $2 \cdot 893$ & $3 \cdot 498$ \\
$\alpha$ & $11 \cdot 866$ & $12 \cdot 549$ \\
$\beta$ &
\end{tabular}

* Due to flooding following torrential rain, we were unable to survey dogs, and thus estimate vaccination coverage, in zone 1.

$\dagger p_{t}(i)=C_{i} * E_{i} * R_{i}$.

f $c 1$ for marked dogs, $c 2$ for unmarked dogs, $\alpha$ and $\beta$ reflect shape parameter of the beta-distribution with mean $=\alpha /(\alpha+\beta)$. 
Table A2. Comparison of the posterior mean estimates of recapture and confinement parameters of the two campaigns: owner charged (new) and free vaccination (previous), respectively*

\begin{tabular}{|c|c|c|c|c|}
\hline & \multicolumn{2}{|l|}{ Zone $2 \dagger$} & \multicolumn{2}{|l|}{ Zone 3} \\
\hline & $\begin{array}{l}\text { Owner charged } \\
\text { (new study) }\end{array}$ & $\begin{array}{l}\text { Free vaccination } \\
\text { (previous study) }\end{array}$ & $\begin{array}{l}\text { Owner charged } \\
\text { (new study) }\end{array}$ & $\begin{array}{l}\text { Free vaccination } \\
\text { (previous study) }\end{array}$ \\
\hline \multicolumn{5}{|c|}{ Recapture probabilities $p_{t}(i) \ddagger$} \\
\hline$t 1$ & $0 \cdot 15(0 \cdot 07-0 \cdot 23)$ & $0 \cdot 29(0 \cdot 22-0 \cdot 38)$ & $0 \cdot 14(0 \cdot 10-0 \cdot 20)$ & $0 \cdot 32(0 \cdot 24-0 \cdot 41)$ \\
\hline$t 2$ & $0 \cdot 22(0 \cdot 10-0 \cdot 33)$ & $0 \cdot 23(0 \cdot 19-0 \cdot 31)$ & $0 \cdot 21(0 \cdot 15-0 \cdot 28)$ & $0 \cdot 29(0 \cdot 23-0 \cdot 37)$ \\
\hline$t 3$ & $0 \cdot 23(0 \cdot 11-0 \cdot 34)$ & $0 \cdot 25(0 \cdot 20-0 \cdot 34)$ & $0 \cdot 22(0 \cdot 16-0 \cdot 30)$ & $0 \cdot 24(0 \cdot 22-0 \cdot 30)$ \\
\hline$t 4$ & $0 \cdot 22(0 \cdot 10-0 \cdot 33)$ & $0 \cdot 22(0 \cdot 19-0 \cdot 29)$ & $0 \cdot 17(0 \cdot 12-0 \cdot 24)$ & $0 \cdot 32(0 \cdot 24-0 \cdot 41)$ \\
\hline \multicolumn{5}{|c|}{ Confinement probabilities of owned dogs§ } \\
\hline$c 1(i) \S$ & $0.53(0 \cdot 22-0 \cdot 73)$ & $0.47(0.47-0.60)$ & $0 \cdot 30(0 \cdot 15-0 \cdot 46)$ & $0 \cdot 48(0 \cdot 37-0 \cdot 60)$ \\
\hline$c 2(i) \S$ & $0 \cdot 15(0 \cdot 03-0 \cdot 36)$ & $0 \cdot 46(0 \cdot 29-0 \cdot 63)$ & $0 \cdot 29(0 \cdot 11-0 \cdot 48)$ & $0 \cdot 38(0 \cdot 22-0 \cdot 55)$ \\
\hline
\end{tabular}

Values in parentheses are $95 \%$ credibility intervals.

* Detailed explanation for parameters see Appendix 1 (available online).

$\dagger$ Transect for the cost campaign not done in zone 1 .

$\$ i=$ zone, $t=$ transect line, higher probability equates higher chance to see the dogs.

$\S c 1$ for marked dogs, $c 2$ for unmarked dogs; higher confinement probabilities equate with better rabies control.

\section{NOTE}

Supplementary material accompanies this paper on the Journal's website (http://journals.cambridge.org/ hyg).

\section{ACKNOWLEDGEMENTS}

We thank all international institutions, collaborators and campaign workers who contributed to this study and ensured its success through teamwork. We also thank the reviewers for their constructive comments that improved the manuscript. We thank the study sponsors: The Swiss Federal Veterinary Office, the Wolfermann-Nägeli Foundation, the Commission for Research Partnership with Developing Countries and the Emilia Guggenheim-Schnurr Foundation. We acknowledge the Swiss National Centre of Competence in Research (NCCR) North-South for co-funding the study supervisor (J.Z.). Merial donated the doses of canine antirabies vaccine, for which we are grateful. The findings and conclusions in this paper are those of the authors and do not necessarily represent the views of the Centers for Disease Control and Prevention.

\section{DECLARATION OF INTEREST}

None.

\section{REFERENCES}

1. Knobel DL, et al. Re-evaluating the burden of rabies in Africa and Asia. Bulletin of the World Health Organization $2005 ; \mathbf{8 3}$ : 360-368.

2. Wandeler AI, et al. The ecology of dogs and canine rabies: a selective review. Revue Scientifique et Technique (International Office of Epizootics) 1993; 12: 51-71.

3. World Health Organization (WHO). WHO expert consultation on rabies. Database (http://www.who.int/ rabies/trs931_\%2006_05.pdf). 2005.

4. Cleaveland $\mathrm{S}$, et al. Estimating human rabies mortality in the United Republic of Tanzania from dog bite injuries. Bulletin of the World Health Organization 2002; 80: 304-310.

5. Babès V. Traite de la Rage [Trekatise on rabies]. Paris: J. V. Baillière et Fils, 1912, pp. 81-119.

6. Willoughby RE Jr, et al. Survival after treatment of rabies with induction of coma. New England Journal of Medicine 2005; 352: 2508-2514.

7. Coleman PG, Dye C. Immunization coverage required to prevent outbreaks of dog rabies. Vaccine 1996; 14: 185-186.

8. Flamand A, et al. Eradication of rabies in Europe. Nature 1992; 360 : 115-116.

9. Zanoni R, et al. Rabies-free status of Switzerland following 30 years of rabies in foxes. Schweizerisches Archiv für Tierheilkunde 2000; 142: 423-429.

10. Cleaveland $\mathbf{S}$, et al. Canine vaccination-providing broader benefits for disease control. Veterinary Microbiology 2006; 117: 43-50.

11. Takayama N. Rabies control in Japan. Japanese Journal of Infectious Diseases 2000; 53: 93-97.

12. Centers for Disease Control and Prevention (CDC). Notice to readers: world rabies day-September 8, 
2007. Morbidity and Mortality Weekly Report 2007; 56: 915.

13. Dürr S, et al. Rabies diagnosis for developing countries. PLoS Neglected Tropical Diseases 2008; 2: 206.

14. Kayali $\mathbf{U}$, et al. Coverage of pilot parenteral vaccination campaign against canine rabies in N'Djamena, Chad. Bulletin of the World Health Organization 2003; 81: 739-744.

15. Kayali U, et al. Cost-description of a pilot parenteral vaccination campaign against rabies in dogs in N'Djamena, Chad. Tropical Medicine \& International Health 2006; 11: 1058-1065.

16. Kaare M, et al. Rabies control in rural Africa: Evaluating strategies for effective domestic dog vaccination. Vaccine 2009; 27 : 152-160.

17. Edelsten RM. Epidemiology and control of rabies in Malawi. Tropical Animal Health and Production 1995; 27: $155-163$.

18. Division of Health Information System. Annual Health Statistics for Chad, 2001 [in French].
19. Mindekem R, et al. Impact of canine demography on rabies transmission in N'Djamena, Chad [in French]. Médicine Tropicale 2005; 65: 53-58.

20. Mindekem R. The geography of risk factors of rabies transmission in urban settings: the case of N'Djaména, Chad [in French] (Dissertation). Montpellier, France: Université Paul-Valery, U.F.R. III, 2003.

21. Caughley G. Petersen estimate. In: Caughley G, ed. Analysis of Vertebrate Populations. New York: J. Wiley \& Sons, 1979, pp. 141-145.

22. Dürr $\mathbf{S}$, et al. Owner valuation of rabies vaccination of dogs, Chad. Emerging Infectious Diseases 2008; 14: 1650-1652.

23. World Health Organization (WHO). National Health Accounts database (http://www.who.int/nha/country/ en/index.html), 2007.

24. Bögel K, Meslin FX. Economics of human and canine rabies elimination: guidelines for programme orientation. Bulletin of the World Health Organization 1990; 68: 281-291. 